\title{
Nuevos Desarrollos en el Enfoque Histórico-Cultural: Su Pertinencia para la Educación Contemporánea
}

\author{
Wanda C. Rodríguez Arocho \\ wandacr@gmail.com \\ https://orcid.org/0000-0002-4460-926X \\ Universidad de Puerto Rico \\ Río Piedras, Puerto Rico
}

Recibido: 11/04/2020 Aceptado: 11/05/2020

\begin{abstract}
Resumen
Desde su formulación original hasta el presente el enfoque histórico-cultural asociado con el trabajo seminal de L.S. Vygotski sobre el origen y desarrollo de las funciones metales superiores ha guardado una estrecha relación con la educación. El propósito de este escrito es examinar el estado actual de esa relación en función de un movimiento revisionista que actualmente hace énfasis en aspectos ignorados o minimizados en las interpretaciones dominantes de la obra de Vygotski. Entre estos aspectos destacan el rol de las emociones, la subjetividad, la situación social del desarrollo, la vivencia y la conciencia en los procesos educativos. Mediante el análisis de textos que se inscriben en ese movimiento revisionista se documenta que éste ha impulsado reflexión crítica en torno a algunos conceptos vygotskianos claves en el área educativa, como es el caso de la zona de desarrollo próximo. Se documenta también que ha propiciado propuestas y prácticas innovadoras como los fondos de conocimiento y los fondos de identidad. Tras la discusión de estas nuevas consideraciones y prácticas, se examina su pertinencia para la educación contemporánea y la formación docente. Se concluye que los desarrollos contemporáneos en el enfoque histórico-cultural pueden contribuir a una educación que responda más efectivamente a las demandas que las transformaciones históricas, sociales y culturales actuales han impuesto sobre ella.
\end{abstract}

Palabras clave: Enfoque histórico-cultural. Vygotski. Educación. Revolución revisionista.

\section{New Developments in the Historical-Cultural Approach: Its Relevance to Contemporary Education}

\begin{abstract}
From its original formulation to the present, the cultural-historical approach associated with the seminal work of L.S. Vygotsky on the origin and development of higher metal functions has been closely related to education. The purpose of this writing is to examine the current state of this relationship in function of a revisionist movement that currently emphasizes ignored or minimized aspects in the dominant interpretations of Vygotsky's work. Among these aspects, the role of emotions, subjectivity, the social situation of development, experience and awareness in educational processes stand out. Through the analysis of texts that subscribe that revisionist movement, it is documented that it has promoted critical reflection about some key Vygotskian concepts in the educational area, such as the zone of proximal development. It is also documented that it has fostered innovative proposals and practices such as knowledge funds and identity funds. After discussion of these new considerations and practices, their relevance to contemporary education and teacher training is examined. It is concluded that contemporary
\end{abstract}


developments in the cultural-historical approach can contribute to an education that responds more effectively to the demands that current historical, social and cultural transformations have imposed on it.

Keywords: Cultural-Historical Approach. Vygotsky. Education. Revisionist Revolution.

\section{Novos desenvolvimentos na abordagem histórico-cultural: Sua Relevância para a Educação Contemporânea}

\section{Resumo}

Desde sua formulação original até o presente, a abordagem histórico-cultural associada ao trabalho seminal de L.S. Vygotski sobre a origem e desenvolvimento das funções mentais superiores tem estado intimamente relacionada à educação. O objetivo deste trabalho é examinar o estado atual dessa relação em termos de um movimento revisionista que atualmente enfatiza aspectos ignorados ou minimizados nas interpretações dominantes do trabalho de Vygotski. Estes aspectos incluem o papel das emoções, da subjetividade, da situação social do desenvolvimento, da experiência e da consciência nos processos educativos. Através da análise dos textos que fazem parte desse movimento revisionista, documenta-se que ele tem promovido uma reflexão crítica sobre alguns conceitos vygotskianos chave na área educacional, como é o caso da zona de desenvolvimento próximo. Também está documentado que tem fomentado propostas e práticas inovadoras, como fundos de conhecimento e fundos de identidade. Após a discussão destas novas considerações e práticas, examina-se a sua relevância para a educação contemporânea e para a formação de professores. $O$ estudo conclui que a evolução contemporânea na abordagem histórico-cultural pode contribuir para uma educação que responda mais efetivamente às demandas que as transformações históricas, sociais e culturais atuais lhe impuseram.

Palavras-chave: Abordagem histórico-cultural. Vygotsky. Educação. Revolução Revisionista.

\section{Introducción}

En su formulación original y en sus expresiones posteriores como enfoque filosófico, teórico y metodológico para el abordar el estudio de la psique, el enfoque histórico-cultural ha otorgado un rol central a la educación en la explicación de las funciones mentales complejas asociadas con la conciencia. El trabajo seminal de L.S. Vygotski, que formalizó este enfoque, ha sido objeto reciente de revisiones que han llevado a cuestionar el énfasis desmedido que se dio a los procesos cognitivos en sus interpretaciones en Occidente y las aplicaciones instrumentales que hizo la educación de éstas. En este artículo realizo un análisis de este asunto. He dividido el tratamiento del tema en cinco partes. En la primera sección ofrezco un trasfondo histórico de la relación entre psicología y educación en el enfoque histórico cultural y examino el movimiento revisionista. En la segunda presento y examino los principales temas que las revisiones actuales han introducido en el campo de los estudios histórico-culturales para adelantar una compresión más profunda y compleja de esa relación. En la tercera parte ilustro 
cómo los temas recuperados en las revisiones, y los elaborados a partir de ellas, se observan en reflexiones críticas, intervenciones e investigaciones psicoeducativas contemporáneas. En la cuarta sección discuto algunas de las implicaciones de los nuevos desarrollos en el enfoque histórico cultural para la práctica y la investigación educativas. Finalizo con una conclusión general.

\section{Trasfondo histórico y movimiento revisionista}

La relación entre educación y psicología fue un tema de interés para L.S. Vygotski desde muy temprano en el desarrollo de su obra. Muestra de ello es un trabajo temprano titulado, Psicología pedagógica: Un curso breve (Vygotski, 1926/2001). Según Blanck (2001), ese texto tenía el propósito de servir de guía a docentes en formación destinados a transformar la educación tradicional prerrevolucionaria con estudiantes de escuela media (10-15 años). El libro, que se fundamenta en experiencias y reflexiones de los años en que Vygotski fue docente, cubre una amplia gama de temas en educación regular y educación especial. A la diversidad temática en este texto subyace el entendido de que si bien la educación parte de la funcionalidad psicológica y los aprendizajes previos de los sujetos, también tiene el potencial de modificar dicha funcionalidad y potenciar aprendizajes y promover desarrollo intelectual.

Vygotski comenzó como su vida profesional como educador y lo fue hasta el final de ésta. En uno de los primeros textos occidentales sobre Vygotski y su obra, Rivière (1984) señala que su producción académica estuvo marcada por tres intereses, aquí mencionados en el orden en que se registran en dicha producción: 1) temas relacionados a "la estética, crítica y promoción institucional del arte", 2) a "cuestiones pedagógicas y educativas", y 3 ) a asuntos "propiamente psicológicos" (p. 18). El entrelazamiento que Vygotski realizó de estos temas en su obra tiene un significado profundo que expresa su concepción de la educación formal como instrumento esencial de la enculturación y humanización' (p. 18). Posteriormente, Vygotski también ponderaría la importancia de la educación informal en propiciar aprendizaje que pueden facilitar, obstaculizar o impugnar los aprendizajes escolares (Vygotski, 1935/2004).

En la idea de interdependencia entre educación y psicología, se unían dos temas relacionados que fueron centrales para Vygotski: el origen social de la conciencia y el desarrollo cultural de la autorregulación de la conducta. Aunque su Psicología pedagógica precede a la formulación de la teoría de Vygotski sobre del origen social de las funciones psíquicas superiores (Vygotski, 1931/1993), y posteriormente revisó algunas de las posiciones expresadas 
en dicho texto (Vygotski, 1934/1997), el texto anticipó la centralidad que ocupa la educación en la psicología de orientación vygotskiana y en el enfoque histórico-cultural.

Cuando la obra de Vygotski fue introducida en Occidente, en el contexto de importantes transformaciones históricas, sociales y culturales (Rodríguez Arocho, 2013, 2015, 2018), el vínculo entre educación y psicología fue uno de los temas que mayor interés captó de miembros de la academia y practicantes en ambos campos. Así se demuestra en obras que marcaron la importancia de esa relación desde temprano en el momento de recepción y apropiación de la obra de Vygotski en Occidente (Álvarez, 1997; Moll, 1990; Daniels, 1993, 2001; Kozulin, 1998). Esto dio paso a un vertiginoso y productivo programa de aplicación de ideas y desarrollo de prácticas educativas que cubrió todos los niveles de educativos, abarcó prácticamente todas las materias enseñada, impactó currículos y sirvió de fundamento conceptual al desarrollo de programas de desarrollo y modificación cognitiva (Chaklin, 2003; Kozulin y Gindis et al, 2003). Según González Rey (2009, 2011a) estos programas partieron de premisas ontológicas y epistemológicas asociadas con el positivismo y el empirismo. Esto también implicaba distorsiones en las ideas metodológicas de Vygotski para la aproximación al objeto de estudio (Rodríguez Arocho, 2011). Aunque las interpretaciones iniciales de la obra de Vygotski siguen teniendo impacto, actualmente coexisten con un movimiento orientado a problematizar esas interpretaciones y promover revisiones a éstas.

El mencionado movimiento aboga por reflexividad histórica tanto del contexto de producción de la obra de Vygotski como de los diferentes contextos en fue recibida y utilizada. Desde el inicio del proceso de recepción algunas voces en la naciente academia vygotskiana se ocuparon de señalar problemas de traducción e interpretación, selectividad en el orden de publicación, descontextualización, falta de consideración a la dimensión filosófica de su obra y sesgos en el manejo de los aspectos ideológicos de ésta (Ageyev, 2003; Bakhurst, 2007; Daniels, 2001; Elhammoumi, 2001; Veresov, 1999). Sin embargo, estos señalamientos y los argumentos con respecto a sus implicaciones en la apropiación de la obra vygotskiana fueron aislados y no tuvieron movilizaron un proceso significativo de reflexividad y autocrítica hasta recientemente (Matusov, 2008).

Ante la situación descrita ha convergido dos movimientos que actualmente impulsan relecturas y reinterpretaciones del legado vygoskiana. El primer movimiento es uno orientado a estudios de recepción e interpretación de la obra de Vygotski fuera de antigua URSS. En 
palabras de uno de sus exponentes, "toda recepción implica desplazamientos, variaciones, omisiones, adiciones y sustituciones" (García, 2019, p. 176). Es de esta forma porque la recepción y apropiación de ideas producidas en otros contextos y tiempos, implican necesariamente actos de lectura que se realizan desde referentes culturales distintos y con propósitos que responden tanto a intereses personales como sociales (Dafermos, 2016).

El segundo movimiento impulsador de nuevas tendencias en las interpretaciones de Vygotski y en el enfoque histórico-cultural es lo que se ha llamado la Revolución Archivística (Yasnitsky, 2010; Zavershneva, 2010a) o Revolución Revisionista (Yastnisky y Van der Veer, 2016). Mediante acceso directo a los archivos de Vygotski facilitado por su familia (Zavershneva 2010ab; Zavershneva y Van der Veer, 2018) se ha iniciado un movimiento que confronta los problemas antes mencionados de forma concertada. Se ha producido un cuerpo de investigación que documenta tanto el contexto y las condiciones de producción como ideas y conceptos desatendidos en actos de recepción y apropiación que privilegiaron la atención al desarrollo de funciones cognitivas por vía de mediaciones pedagógicas fundamentalmente instrumentales (González Rey, 2011a). Estas producciones destacan temas omitidos o minimizados en el tratamiento de la obra de Vygotski hasta recientemente. Entre éstos se encuentran la situación social del desarrollo, las emociones, la vivencia, la conciencia, la perspectiva dialéctica y el enfoque sistémico representado en su noción de unidades de análisis. La inclusión de estos temas en las aproximaciones actuales a la obra de Vygotski ha generado un movimiento hacia la complejidad en la explicación de la psique que ha tenido implicaciones y generado aplicaciones en la integración de psicología y educación.

\section{Temas contemporáneos}

Varios textos recientes se ocupan en detalle de los temas recién mencionados (Dafermos, 2018; Fleer, González Rey y Veresov, 2017; González Rey, Mitjáns Martínez y Magalhães Goulart, 2019). En una crítica a la académica vygotskiana, Matusov (2004) destacó que, en términos generales, mostraba muy poca reflexividad sobre el contexto de sus propias producciones y fallaba en hacer explícitos los argumentos que dichas producciones cuestionaban o refutaban. Los desarrollos contemporáneos enfrentan frontalmente ambas críticas. En primer lugar, reconocen transformaciones históricas, sociales y culturales que han impacto concepciones y prácticas en la ciencia, relaciones con la tecnología y los ambientes vitales y visiones de mundo. En este sentido, en estas producciones abundan las reflexiones históricas, 
filosóficas y sociológicas sobre la ciencia en general y las disciplinas particulares. Se separan explícitamente del positivismo y el empirismo y rechazan la simplificación de lo complejo. En segundo lugar, tienden identificar los autores y las autoras a quienes interpelan en construcciones dialógicas. Se caracterizan por utilizar la problematización y la indagación dialógica como herramientas de análisis (Rodríguez Arocho, 2015) y precisar los fundamentos que cuestionan y los que defienden.

El texto editado por Fleer, González Rey y Veresov (2017) que trata los temas de vivencia (perezhivanie), emociones y subjetividad es excelente ejemplo de lo descrito. Dicho texto contiene 12 capítulos que tratan aspectos teóricos y metodológicos sobre estos temas e inicia con una declaración de lo que persigue su abordaje a los temas ignorados o minimizados en el tratamiento de la obra vygotskiana. La declaración articula tres principios fundamentales: 1) trascender el determinismo social, 2) superar la concepción de la mente como el resultado de procesos de interiorización y 3) promover una perspectiva sistémica caracterizada por la dialéctica y la complejidad. Estos principios se expresan como respuesta contestaria a interpretaciones de Vygotski que acentuaron la fuerza de la mediación social restando importancia o ignorado una fuerza generadora en el sujeto que crear sentidos propios diferentes a los intencionados o significados por otros en esa mediación.

González Rey (2011a) argumenta que la situación descrita se explica porque en la recepción de la obra de Vygotski en Occidente dio énfasis su obra intermedia, la cual se enfocó en el estudio de lo que él llamó funciones psíquicas superiores, entre las que incluyó la atención voluntaria, la memoria lógica, el razonamiento y el ejercicio de la voluntad (Vygotski,1931/1995). Al formular la ley genética general del desarrollo cultural o la ley de la doble formación, Vygotski planteó que estas funciones se originan en actividades entre la gente (interpsicológicas) y luego de un proceso de interiorización se transforman en funciones mentales individuales (intrapsicológicas).

Vygotski argumentó que la educación puede jugar un rol fundamental en esa transformación, idea que fue fundamental en la interpretación que se dio a su concepto de zona de desarrollo próximo (zdp). Sin embargo, es posible argumentar que en la apropiación de ambas ideas para orientar programas educativos la interpretación fue sesgada por un momento en que el cognitivismo se establecía como un modelo dominante en las explicaciones psicológicas (Rodríguez Arocho, 2000). Por ejemplo, al citar la ley de genética general del 
desarrollo cultural, Vygotski afirma que el paso de lo externo a lo interno “...modifica el propio proceso, transforma su estructura y sus funciones" (Vygotski, 1931/1995, p. 150). Es decir, no lo considero un proceso de copia automático de copia. De igual modo, concibió la idea de zona de desarrollo próximo hacia el final de su vida, cuando también elaboró las ideas de situación social del desarrollo y vivencia, que permiten una comprensión más compleja de la zdp que la reducida tradicionalmente a la idea de andamiaje (Rodríguez Arocho, 2015). Estas ideas se han convertido en temas centrales en las explicaciones e intervenciones educativas que se generan en la actualidad desde la perspectiva histórico-cultural.

Hacia el final de su vida, Vygotski retomó temas que había trabajado en sus obras iniciales sobre arte (del Río y Álvarez, 2007) y educación creativa (Álvarez y del Río, 2007). Entre esos temas se encuentran las emociones, la imaginación, la fantasía y el arte como psicotecnia del sentimiento. Elaboró sobre estos temas en sus trabajos finales (Vygtoski, 1934,1935) en un esfuerzo inconcluso de integrarlos en un sistema conceptual. Algunos trabajos contemporáneos enfocan estos temas, mientras que otros realizan elaboraciones teóricas y metodológicas a partir de ellos y persiguen su avance dentro de la perspectiva histórico-cultural (Fleer, González Rey y Veresov, 2017; González Rey, Mitjáns Martínez y Magalhães Goulart, 2019).

\section{Emociones y afectividad}

La forma en que el lugar de las emociones en la teoría de Vygotski fue desatendido en las interpretaciones de su obra que privilegiaron la actividad cognitiva en el desarrollo humano ha sido objeto de análisis recientemente (Rodríguez Arocho, 2015). Esta actividad cognitiva se orientó principalmente a la relación entre el lenguaje y pensamiento, particularmente el desarrollo de conceptos científicos, abstracciones y generalizaciones mediadas por el lenguaje (Karpov, 2014). Sin embargo, hay múltiples instancias en la obra de Vygotski donde se plantea un problema fundamental en esa orientación. Destaca, por ejemplo, su denuncia:

La primera cuestión que se plantea cuando hablamos de la relación entre pensamiento y lenguaje y los restantes aspectos de la conciencia es la de la conexión entre el intelecto y el afecto. Como es sabido, la separación entre el aspecto intelectual de nuestra conciencia y su aspecto afectivo, volitivo, constituye uno de los defectos básicos más graves de toda la psicología tradicional (Vygotski, 1934b, p. 24).

A esta denuncia sigue la argumentación de que entender la conciencia como “...un flujo autónomo de ideas que se piensan a sí mismas” es desacertado porque la segrega de la plenitud 
de la vida, de los impulsos, los intereses y las inclinaciones vitales del sujeto que piensa" no permitirá entenderla y explicarla científicamente. Argumenta que esas dos tareas requieren enfocarse en unidades de análisis y no en elementos aislados pues cuando se hace de ese modo se "revela la existencia de un sistema semántico dinámico, representado por la unidad de los procesos afectivos e intelectuales" (Vygotski, 1934b, p. 24).

La centralidad otorgada a las emociones en estas dos citas, que provienen del primer capítulo de su obra Pensamiento y lenguaje, Vygotski, 1934b), es recalcada con fuerza en el capítulo final esa obra cuando plantea que:

Nos queda el último y definitivo paso en el análisis de los plantos internos del pensamiento verbal. El pensamiento no es la última instancia en este proceso. El pensamiento no nace de si mimos ni de otros pensamientos, sino de la esfera motivacional de nuestra conciencia, que abarca nuestras inclinaciones y nuestras necesidades, nuestros intereses e impulsos, nuestros afectos y emociones. Detrás de cada pensamiento hay una tendencia afectivo-volitiva. Sólo ella tiene la respuesta al último por qué en el análisis del proceso de pensar (p. 342).

Al considerar el lugar de las emociones en la teoría de Vygotski hay que tomar en consideración varios asuntos. En primer lugar, partía del supuesto de que igual que la cognición, las emociones tiene origen sociocultural y se desarrollan en contextos de actividad vital. En su crítica al biologicismo y naturalismo que caracterizaba las teorías de emociones dominantes en su época, Vygotski (1935/2004) planteó que las emociones no pueden entenderse al margen de la dinámica de la vida humana y que es desde los procesos involucrados esa dicha dinámica que cobran sentido y significado para el sujeto. En segundo lugar, entendía que tanto esa dinámica como los procesos que la inciden operan bajo los principios de la dialéctica.

\section{Dialéctica}

A pesar de la recurrente referencia a esos principios en su obra, Dafermos (2018) plantea que la dialéctica ocupó un lugar central en la producción de Vygotski y en el desarrollo de la perspectiva histórico-cultural que ha sido mayormente ignorado en las interpretaciones de su obra en Occidente. Elabora argumentos para recuperar ese aspecto de su legado. Documenta la forma en que Vygotski (1927/1991) asumió la dialéctica como orientación filosófica, aceptando sus premisas ontológicas y epistemológicas. La conceptuación de la realidad como producto de fuerzas internas y externas en tensión y de contradicciones entre lo general y lo particular, lo conceto y lo abstracto, lo aparente y esencial, le llevaron a concluir que había que considerarlas 
como las fuerza que impulsan el desarrollo humano (Dafermos, 2018). Fleer, González Rey y Veresov (2017) argumentan que la idea de Vygotski de una relación dialéctica entre el funcionamiento interpsicológico y el intrapsicológico que las emociones se construyen en relaciones enmarcadas culturalmente. Argumentan que la perspectiva relacional dialéctica permite rebasar el determinismo social y entender la dinámica entre sentimientos y emociones como expresiones que son culturalmente interpretadas y vividas.

La reivindicación de la dialéctica en estos momentos busca interpelar los modelos tradicionales de desarrollo como una secuencia ordenada de etapas predecibles, inalterables y universales. Este razonamiento se expresa en otros tres temas rescatados del legado de Vygotski, situación social del desarrollo, vivencia y conciencia (Rodríguez Arocho. 2010, 2018), y de un tema de reciente elaboración, la subjetividad (González Rey, 2011b).

\section{Perzhivanie (Vivencia)}

En la actualidad se está utilizando la palabra rusa perzhivanie en textos en otros idiomas porque hay dificultad con una traducción literal del término. En la edición de las obras escogidas de Vygotski en inglés el término fue traducido como "experiencia", mientras que en las obras escogidas en español fue traducido como "experiencia atribuida de sentido" y como "vivencia" (Rodríguez Arocho, 2010). González Rey y Mitjáns Martínez (2016), Mok (2017) y Veresov (2017) analizan en detalle la historia del concepto y su lugar en el sistema teórico que Vygotski refinaba hacia el final de su vida. Estos trabajos destacan que Vygotski utilizó el concepto por primera vez en una de sus obras más tempranas, Psicología del arte. En esa instancia la relacionó con otros conceptos de la dimensión afectiva individual como la imaginación, la fantasía y las emociones. Retomó el concepto varias de sus obras finales El problema de la edad (Vygotski, 1932) y La crisis de los siete años (Vygotski,1933) y El problema del ambiente (Vygotski, 1935b). En estos trabajos declaró que la perzhivanie debía ser la unidad de análisis para abordar el estudio de la personalidad. Según González Rey y Mitjáns Martínez (2016), Vygotski trabajaba en una representación del domino afectivo como "un sistema complejo de la psique humana, el centro del cual era la perzhivanie o la experiencia emocional atribuida de sentido subjetivo (p.145). Aunque Vygotski no llegó a elaborar con respecto a la relación entre vivencia y sentido subjetivo, la misma fue importante en la obra de Lydia Bozhovich (González Rey, 2019a) y también lo ha sido en la formulación de la teoría de una teoría de la subjetividad en el enfoque histórico-cultural por González Rey (2019b). 


\section{Situación social del desarrollo}

El tratamiento que Vygotski $(1932,1933,1935)$ dio a las emociones y a la vivencia en sus últimos trabajos implica una concepción diferente del ambiente a la que ha predominado en la psicología tradicional, en la cual el ambiente se considera algo externo al sujeto que es posible describir en índices objetivos absolutos y explicar en término de relaciones causales lineales. Argumentó que esta forma de entender la relación entre sujeto y ambiente presentaba problemas teóricos y metodológicos a la psicología. Propuso que es relación es dinámica, dialéctica y cambiante, por lo que no puede comprenderse sin entender las vivencias que las configuran en particulares situaciones de desarrollo. Planteó que las relaciones con el ambiente, particularmente con el ambiente social, cambian en el curso del desarrollo humano y son peculiares, específicas, únicas e irrepetibles y constituyen la situación social de desarrollo cada sujeto. Es en esa situación que las vivencias se configuran y adquieren sentido para el sujeto. Por lo tanto, no estudiar esta relación interna del sujeto con las otras personas en su entorno equivale a ignorar que es un participante activo en su propia situación de desarrollo.

Enfrentar este problema implica estudiar, ante todo, cómo el sujeto significa y siente su situación. Esta unidad entre el significado y el sentido, el intelecto y el afecto fue cobrando fuerza en los últimos textos de Vygotski y termina con la introducción "de la vivencia como unidad de conciencia, es decir, como unidad en la cual las propiedades básicas de la conciencia figuran como tales, mientras que, en la atención, en el pensamiento, no se da esa relación” (p. Vygotski, 1933, p.383). El planteamiento es que la atención y el pensamiento son elementos de la conciencia, pero que ésta no se reduce a ellos ni ellos pueden por sí solos explicarla. Hacia el final de su vida Vygotski, consideraba la vivencia o perzehivanie era la base de la conciencia y la fuerza motriz del desarrollo.

\section{Subjetividad}

La teoría de la subjetividad formulado por González Rey (2002, 2011b), dentro del marco conceptual del enfoque histórico-cultural, elabora sobre las ideas antes presentadas. Esta teoría ha generado interés en la académica vygotskiana contemporánea (Fleer, González Rey y Veresov, 2017; González Rey, Mitjáns Martínez y Magalhães Goulart, 2019). La subjetividad es entendida como un nuevo domino ontológico de los fenómenos humanos, individuales o sociales, que es inseparable del mundo sociocultural en que los seres humanos viven sus experiencias (González Rey, 2019b). Elabora cinco argumentos para sostener esa definición. 
El primer argumento es que la cultura posibilita cambios psicológicos y generacionales al ser un sistema compartido de producciones simbólicas “que se objetivan en el lenguaje, las prácticas discursivas, las representaciones sociales, los mitos, los sistemas normativos, las religiones y otras producciones culturales" (p. 182). Dado que las emociones están imbricadas en estos procesos simbólicos, "la subjetividad se torna en un componente intrínseco de la cultura" (p. 182).

El segundo argumento para sostener que la subjetividad es un nuevo domino ontológico es que ésta no es un fenómeno intrapsíquico sino "una cualidad característica de cada proceso, realidad y sistema humano" (p. 182) que integra lo individual y lo social. El tercer argumento se apoya en las transformaciones de la historia sociocultural. Cada generación adviene a un mundo cuya organización cultural da forma a imaginarios que se consideran realidades objetivas, pero que cambiarán radicalmente para la generación siguiente resultando en otros imaginarios y otras realidades. Ejemplo de esto son las transformaciones psíquicas relacionadas a las tecnologías (Rodríguez Arocho, 2018). El cuarto argumento acentúa la relación entre lo histórico-sociocultural y lo psicológico al plantear que, aunque los miembros de cada generación comparten una cultura, cada individuo, grupo e institución la vive de diferente forma. Este argumento recalca que las experiencias vividas no son reflejo o asimilación de elementos en una realidad objetiva sino producciones de sentido en que intelecto y afecto se unen. Finalmente, esa unidad revela complejas producciones subjetivas o configuraciones en las que, como humanos, generamos sentidos apoyados en experiencias a lo largo de la vida en los múltiples contextos en que ésta trascurre. En ese transcurso, las relaciones sociales de carácter simbólicoafectivo viabilizan la emergencia y expresión de nuestras emociones y nuestros motivos.

El planteamiento de un domino ontológico como la subjetividad, según descrita, implica la necesidad una nueva epistemología, pues el nuevo objeto de estudio resiste el reduccionismo, la universalidad de la métrica y las explicaciones casuales lineales. Por ello, para el estudio de la subjetividad desde la perspectiva descrita González Rey propuso una epistemología cualitativa orientada por un método constructivo-interpretativo (González Rey, 2107; González Rey y Mitjáns, 2019). Las estrategias de este enfoque descansan principalmente en el diálogo, la comunicación y la exploración colaborativa de significados y sentidos, tanto en la práctica profesional como en la investigación. 


\section{Conciencia}

La propuesta metodológica antes mencionada para estudiar la subjetividad busca rebasar los límites que los métodos tradicionales impusieron a loa psicología y que Vygotski denunció muy temprano en su producción científica un problema fundamental. En sus palabras:

Nuestra literatura científica elude insistente e intencionalmente el problema de la naturaleza psicológica de la conciencia, y trata de no darse cuenta de él, como si para la nueva psicología no existiese en absoluto. [...] Al ignorar el problema de la conciencia, la psicología se está cerrando a si misma el camino de la investigación de problemas más o menos complejos del comportamiento humano (Vygotski, 1925/1991, p.39).

Al final de su obra Vygotski retoma este tema y plantea la clave para abordar comprender la conciencia requiere entender la relación entre lenguaje y pensamiento en el curso del desarrollo humano y sus transformaciones. Los argumentos para sostener este planteamiento son sintetizados en el capítulo final de Pensamiento y lenguaje (Vygotski, 1934), donde no sólo subraya la idea de unidad entre intelecto y afecto, sino que introduce (sin articularla de este modo) la dimensión subjetiva y la elaboración de sentido. Plantea que para comprender el lenguaje ajeno no basta conocer las palabras, sino que hay intentar conocer el pensamiento de quien las expresas. Argumenta que la comprensión ese pensamiento requiere conocer los motivos (causas), intenciones (propósitos), afectos y emociones del sujeto que lo expresa. En este texto Vygotski también señala el pensamiento (entendido de esta forma compleja) y el lenguaje como aspectos de la conciencia, junto con otros que no especifica, pero que a base de los escritos de ese mismo tiempo puede inferirse que incluyen la perezhivanie o vivencia.

El estudio de los archivos de Vygotski y sus notas personales ha llevado a Zavershneva (2010ab) a plantear que los brevemente reseñado en el párrafo anterior puede entenderse como un prolegómeno o tratado introductorio de los fundamentos centrales que habría de elaborar en su siguiente trabajo. Se especula que ese trabajo era una teoría psicológica general que incluía con la conciencia como dimensión central. Esta es una idea compartida por Dafermos (2018), quien plantea que el trabajo inacabado de Vygotski buscaba integrar en un sistema dinámicos los temas examinados en esta sección: emociones, volición, vivencias, significado y sentido, campos semánticos, la relación entre pensamiento y lenguaje, la actividad psicofísica, y la desintegración de sistemas psicológicos en patologías como la esquizofrenia. La noción de sistema podría ofrecer un abordaje dialéctico, holístico e histórico-cultural y complejo al estudio 
de la conciencia. Por supuesto, tal abordaje exigiría métodos de estudio diferentes a los tradicionales. Zavershneva (2016) argumenta que este concepto de conciencia es más complejo que de funciones psíquicas superiores identificado con el legado de Vygotski y que supera el dualismo que distingue y separa éstas de las funciones psíquicas elementales.

En resumen, los temas contemporáneos presentan una perspectiva compleja en que el sujeto y su situación social de desarrollo cobran un interés particular y donde las emociones, las vivencias, las interacciones sociales, la comunicación y la se entrelazan a la hora de explicar la conducta humana. Siguiendo la tradición vygotskiana de entrelazar psicología y educación, se ha producido un cuerpo trabajo de investigación e intervenciones en el campo educativo que incorpora los temas contemporáneos examinados.

\section{Reflexiones teóricas, intervenciones e investigaciones actuales}

Las reflexiones teóricas intervenciones e investigaciones actuales que examino a continuación se caracterizan por el uso de uno o más de los conceptos examinados en apropiaciones creativas que fortalecen el enfoque histórico cultural. He seleccionado tres aportes que me parecen particularmente relevantes porque, además de solidez teórica y producciones investigativas interesantes, son evidencia de que es posible realizar trabajo académico de excelencia al tiempo que se asumen posiciones ético-políticas. Este es un asunto que la psicología tradicional ha ignorado o evadido tanto como ha evadido el debate ontológico y epistemológico (González Rey, 2009). Esa coincidencia no es fortuita. La proclamación de separación tajante entre filosofía y ciencia que ha marcado el discurso psicológico ha sido un lastre en el desarrollo de aproximaciones complejas a la conducta propiamente humana. Al ser la ética una rama de filosofía y al construirse un imaginario de ciencia como actividad neutral, tampoco ha habido mucho especio para ella fuera de las corrientes marginales en la psicología y en la educación.

Sin embargo, el tema del posicionamiento ético-político en las construcciones y usos del conocimiento psicológico ha sido central en movimientos como la psicología de la liberación, la pedagogía y la psicología críticas, los tres con importantes aportes desde América Latina (Rodríguez Arocho, 2010). Sin embargo, no es hasta recientemente que se observa un esfuerzo sistemático por provocar y propiciar diálogo entre estos movimientos (Fleer, González Rey y Jones, en imprenta). Ese diálogo guarda la promesa de dar un lugar a la dimensión ético-política en las producciones de la psicología histórico-cultural en el área educativa y puede beneficiarse 
con el reconocimiento de esfuerzos específicos por reconocer su importancia y actuar en consecuencia. Tres de esos esfuerzos son las reflexiones críticas sobre sujeto, situación y educabilidad de Ricardo Baquero (2017) y los conceptos de fondos de conocimiento y fondos de identidad desarrollados por Luis C. Moll y Moisés Esteban-Guitart (Estaban-Guitart y Moll, 2014ab) con colaboraciones diversas en sus respectivos equipos de investigación.

\section{Sujeto, situación y educabilidad}

De los tres autores mencionados, Baquero (2001, 2003, 2006, 2009, 2017) ha sido el más frontal en su crítica a la falta de reflexividad en psicología y educación en torno a los supuestos filosóficos y las consecuencias ético-políticas de las prácticas sociales que atraviesan la educación en general y las prácticas pedagógicas concretas en las aulas. Ha sido consecuente en plantear el rol desempeñado por la psicología y la educación al abordar como productos de la naturaleza propiedades, cualidades y procesos humanos que fueran hechos de la naturaleza y no de la actividad sociocultural. En particular, ha analizado cómo los ideales de regularidad, progreso y universalidad asociados con la Modernidad sirvieron para el desarrollo de producciones en psicología y aplicaciones en educación que asumieron en sus discursos y prácticas. Estos ideales subyacen a todas las teorías de etapas de desarrollo que han sido traducidas a abordajes y prácticas educativas. Su unión con el ideal racionalista para orientar los procesos de escolarización masiva que implicaban un diseño particular de la mente como reproductora de formas y contenidos. Se trata de un diseño marcado por la uniformidad con poco o ningún espacio para considerar la variabilidad en las formas de aprendizaje de los sujetos en la didáctica y en la evaluación. Ese diseño tiene consecuencias significativas en la vida de los sujetos. Baquero $(2001,2003)$ ha abordado con a profundidad dos asuntos relacionados a esas consecuencias. El primero trata de la sospecha en torno a la posibilidad de una educación efectiva para ciertos sujetos. El segundo asunto trata de la educación como práctica socioinstitucional que impacta la configuración de la subjetividad.

Baquero $(2001,2003)$ subraya la dimensión ética-política antes mencionada al examinar problemas de inequidad educativa y justicia social. Plantea que "la pregunta por la posibilidad y el alcance de lo educativo cobra dramatismo muy cruel en la atención, por supuesto, de los alumnos provenientes de los crecientemente castigados sectores populares" (Baquero, 2001, p. 71). En situaciones sociales de desarrollo caracterizadas por la precariedad, donde hay múltiples carencias, la escolarización y los procesos de enseñanza y aprendizaje implicados se practican 
de forma descontextualizada e irreflexiva. El aparato escolar mismo pone en duda la posibilidad de que esos procesos ocurran exitosamente. Este autor señala que esa duda o sospecha parece "razonable" en virtud de "los evidentes índices de repitencia, deserción o de ingreso tardío al sistema, como por la creciente sospecha de que la baja en los logros a que aspiramos muchas veces institucionalmente se traduce incluso en la promoción en ciertos casos de los alumnos a pesar de muchas veces no haber obtenido siquiera los aprendizajes esperados en un contexto de expectativas ya reducido" (p.72).

Es pertinente evocar aquí la preocupación expresada por Vygotski (1935) enfocar en los indicadores o índices que objetivan una realidad que es diferente a las vivencias del sujeto en su particular situación de desarrollo. En la consideración de este problema Vygotski fue enfático al subrayar la necesidad de abordarlo como unidad y no segmentado en índices. Baquero (2017) avala ese énfasis y plantea la necesidad de abordar las relaciones entre los sujetos y sus situaciones sociales de desarrollo como la unidad para entender y explicar los procesos de aprendizaje y desarrollo. Dicha unidad es sistémica, dialéctica y compleja y no puede reducirse a sus componentes. Desde esta perspectiva, la repetición de grados, la llamada deserción escolar y el ingreso son fenómenos históricamente situados, culturalmente mediados y encarnados en prácticas sociales que generan tanto configuraciones de sentido subjetivo como prácticas educativas y acción social. Este ha sido un tema central en algunos acercamientos socioculturales al problema de la relación aprendizaje, desarrollo y evaluación en contextos escolares (Rodríguez Arocho, 2010) y al problema de los criterios que se utilizan para definir y valorar la excelencia académica y la calidad educativa (Rodríguez Arocho, 2011).

Además de la sospecha desde la que parte la práctica escolar institucionalizada expresada en documentos de política educativa, diseño curricular y prácticas didácticas que suscriben los ideales de regularidad, progreso, universalidad y racionalidad, la persona es colocada en frente a actividades en el aula que juegan un papel importante en su particular configuración subjetiva. Baquero (2017) elabora sobre la relación entre prácticas educativas y subjetividad: “...los procesos de subjetivación no pueden escindirse de cierto poder perfomativo de las prácticas instituidas subjetivantes y creadoras de estándares de los cuales apropiarse, recrearlos o rechazarlos" (p.293). Esta es una consideración importante en la que se reconoce el carácter generador de un sujeto que es capaz de apropiarse, recrear, rechazar y crear nuevas 
condiciones de posibilidad. Esas acciones pueden entenderse desde la noción de conciencia como sistema que se configura en la situación social de desarrollo y las vivencias del sujeto.

La idea de unidad de análisis es central en estas reflexiones. También lo ha sido en el desarrollo de los conceptos de fondos de conocimiento y fondos de identidad. Estos constituyen expansiones o elaboraciones de algunos de los temas contemporáneos antes discutidos y rebasan la reducción de los procesos de aprendizaje y desarrollo a un individuo autocontenido y autónomo. Igualmente presenta una visión de las prácticas educativas que supera el determinismo social.

\section{Fondos de conocimiento}

En la introducción a su conocido libro Vygotsky and education: Instructional implications and applications of socio-historical psychology, Moll (1990) destacó que para Vygotski la educación es la quintaesencia de la mediación cultural. Los trabajos en ese libro se orientan a argumentar teóricamente y mediante investigaciones que el desarrollo psicológico de los sujetos depende de los procesos de enseñanza-aprendizaje, que esos procesos son tanto formales como informales, que están situados históricamente y mediados socioculturalmente y que en él se integran lo cognitivo y lo afectivo. Aunque en el momento en que se produce esta obra los temas contemporáneos no eran centrales en las producciones de los enfoques históricoculturales o socioculturales, algunas propuestas para elaboraciones más complejas en los abordajes a la educación fueron anticipadas.

La idea de que la educación es central en el desarrollo de procesos cognitivos, de formas de conciencia sobre el mundo y nuestra relación con él se aprecia de varias maneras en la obra de Moll y colegas. Deriva de sus planteamientos que la personalidad se configura en el curso de vivencias y experiencias de aprendizaje (formales e informales) en particulares situaciones de desarrollo que son inseparables de las condiciones de vida del sujeto. Esta concepción es central en el concepto de fondos de conocimiento. Aunque éste se asocia principalmente con el trabajo de Moll y su equipo de investigación (González, Moll y Amanti, 2005; Moll, 1990, 1997, 2011, 2014), el propio Moll reconoce su origen en las investigaciones de Vélez-Ibáñez y Greenberg (1990). En estas investigaciones antropológicas en hogares en la frontera entre Estados Unidos

y México fueron identificadas estrategias y prácticas culturales en las que se compartían e intercambian información y destrezas, que estos investigadores denominaron fondos de conocimiento. El aporte de Moll y colegas consistió en transferir a la educación el concepto e 
inscribirlo conceptualmente en el enfoque sociocultural. Trabajando con la misma población en el estado de Arizona desarrollaron estudios etnográficos en los que exploraron las expresiones de esos fondos de conocimiento en la escuela y su relación con prácticas educativas concretas. En estos estudios el foco no es el estudiante como individuo sino como miembro de una familia y de una comunidad escolar. Las relaciones sociales y las interacciones personales en las aulas y en el hogar reciben un lugar central en el examen de procesos de aprendizaje y desarrollo.

Moll (1997) define los fondos de conocimiento como "cuerpos de conocimiento culturalmente desarrollados e históricamente acumulados y destrezas esenciales para el funcionamiento y bienestar familiar o individual" (p.47). Uno de los logros más relevantes del programa de investigación desarrollado a partir de este concepto ha sido, por un lado, el reconocimiento de la situación social del desarrollo y las vivencias de los sujetos que aprenden y, por otro lado, la legitimación de los saberes no académicos. En la práctica educativa esto implica una aproximación a la dinámica entre conceptos espontáneos y conceptos científicos de mayor riqueza y complejidad que las aproximaciones tradicionales que obvian la relación dialéctica entre éstos. La dicotomía tajante entre ambos se quiebra y se abren oportunidades para apreciar cogniciones distribuidas y acciones colaborativas en ambientes que en ocasiones son adversos al desarrollo porque subestiman saberes y quehaceres no escolarizados.

La aproximación teórica y metodológica de Moll y colegas a los fondos de conocimiento implica también un examen a la cultura, no como abstracción, sino como experiencia vivida. Al integrar docentes a la investigación y relacionarlos con "la cultura vivida o la cultura en acción" (p. 48) en sus hogares se hace posible el uso de esos fondos de conocimiento para facilitar el éxito escolar, principalmente en situaciones en que la cultura escolar presenta diferencias abismales con la cotidianidad y puede obstaculizar procesos de desarrollo. La cultura vivida y las vivencias experimentadas por familias inmigrantes y de bajo nivel socioeconómico colocan su educabilidad bajo sospecha (Baquero, 2007) pues se ha demostrado que los hijos e hijas de familias con esas características enfrentan grandes obstáculos para alcanzar éxito escolar y presentan altas de “fracaso' escolar.

En el tratamiento de doble problema el programa de investigación de fondos de conocimiento ha asumido su posicionamiento ético-político. Por un lado, ha asumido el 
compromiso de crear oportunidades para que el aprendizaje en la escuela se vincule con las vivencias en el hogar y en la comunidad. Por otro lado, ha avanzado la idea de que el proceso de construcción de conocimiento no es uno reproductivo, sino que implica transformaciones psíquicas cognitivas y afectivas en el sujeto. Para Moll (1997) "la creación cultural, mediatizadora, más importante en el desarrollo del niño es, precisamente, el niño” (p.49). Se reconoce de este modo al sujeto como una fuerza generadora en su propia constitución.

La elaboración del concepto de fondos de conocimiento descrita sirvió de fundamento al desarrollo del concepto de fondos de identidad propuesto por Esteban-Guitart (2016), el cual examino a continuación.

\section{Fondos de identidad}

La presentación más completa de la noción de fondos de identidad es la de EstebanGuitart en 2016. Sin embargo, la misma fue adelantada en 2014 por Esteban-Guitart y Moll (2014ab). En dos artículos publicados declaran que el concepto de fondos de identidad se desarrolla a partir del concepto de fondos de conocimiento y se discuten investigaciones sobre ambos que se caracterizan por aproximaciones principalmente cualitativas en su metodológicas. Muchas de las estrategias de investigación utilizadas sincronizan con la propuesta de epistemología cualitativa propuesta por González Rey (2007) para el estudio de la subjetividad. Esto no es de extrañar pues ambos conceptos dialogan desde su fundamentación teórica y metodológica (Rodríguez Arocho, en imprenta). Generalmente utilizan estrategias que implican diálogo y actividades creativas donde la imaginación y la fantasía tienen un espacio que facilita la comunicación entre estudiantes, docentes e investigadores en la negociación de significados y la construcción de conocimiento.

El concepto de fondos de conocimiento hizo posible visibilizar y valorar los artefactos culturales producidos y utilizados en hogares y comunidades. Esto le otorga un lugar central a la cultura vivida atravesada por vivencias y reclama su consideración como dimensiones que pueden y deben integrarse a la experiencia escolar para hacerla más pertinente y significativa, para atribuirle sentido. Desde ese punto de partida el concepto de fondos de identidad se orienta a explicar cómo ocurre ese proceso mediante el cual los sujetos le otorgan sentido a esas vivencias para definirse, expresarse y comprenderse. Al definir el concepto, Esteban Guitart y Moll, 2014a) enuncian: “Utilizamos el término fondos de identidad para referirnos a los recursos 
históricamente acumulados, desarrollados culturalmente y socialmente distribuidos que son esenciales para la autodefinición, autoexpresión y autocomprensión de una persona” (p.31).

Para entender y explicar la identidad es necesario conocer las condiciones históricas, sociales y culturales en que se configura y cómo son vividas por el sujeto. En otras palabras, hay que enfocar a los sujetos del aprendizaje y las formas de subjetivación que se dan en la escuela pero que la trascienden. Esta forma de abordar el problema presupone que la identidad, independientemente de su pluralidad de definiciones (Esteban-Guitart \& Moll, 2014a) no es una entidad metafísica. La identidad, como la subjetividad y la conciencia, no son fenómenos comprensibles al margen de las acciones que realizan los sujetos, de su historia-cultural, de sus interacciones sociales y de las redes simbólico-emocionales que emergen y se configuran en el curso del desarrollo humano. En el diálogo entre estos conceptos aparece la centralidad de la idea de Vygotski de la unidad sujeto-ambiente, la situación social del desarrollo y experiencia emocional atribuida de sentido subjetivo. Estos conceptos son inclusivos y se inscriben en una visión dialéctica, compleja y sistémica en la que Vygotski trabajaba hacia el final de su vida.

En su elaboración conceptual Esteban Guitart y Moll (2014ab) plantean que los fondos de conocimiento consisten en objetos y relaciones en la cultura vivida que se transforman en fondos de identidad cuando los sujetos se apropian de ellos y los integran a su sistema psicológico. La construcción del concepto de fondos de identidad descansa en análisis crítico de resultados de investigaciones. En ese proceso se notó que, pese a que se identificaban fondos de conocimiento en los hogares y se incorporaban a las lecciones escolares concretas, ello no siempre resultaba en la atribución de significado y sentido a la experiencia de aprendizaje o en una mejoría en la situación escolar (Esteban-Guitart \& Saubich, 2013). Para que el aprendizaje fuera significativo y tuviera sentido debía darse una apropiación de los fondos de conocimiento por parte del sujeto y su transformación en fondos de identidad. Estos fondos, de origen social y cultural, son una expresión del sentido subjetivo que se elabora desde experiencias previas y en la propia situación de aprendizaje. En este sentido, demuestran la unidad entre afecto y cognición antes examinada.

El programa de investigación desarrollado por Esteban Guitart y colegas les ha permitido la identificación de intereses, experiencias y conocimientos integrados en la forma en que una persona se refiere a sí misma. Esto les ha llevado a la conclusión de que hay una variedad de fondos de identidad, entre los que se mencionan fondos geográficos, fondos culturales, fondos 
sociales, fondos institucionales y prácticas de identidad. Cada uno de estos fondos exige poner en movimiento fondos de conocimiento, pero sólo cuando son significados y expresados como señas identitarias que pasan a ser fondos de identidad. Estas formas de identidad ponen de manifiesto, una vez más, la importancia de la situación social del desarrollo y las vivencias que viabiliza.

En síntesis, la recuperación de temas ignorados o minimizados en las interpretaciones originales y dominantes de la obra de Vygotski ha viabilizado reflexiones teóricas que enfatizan y amplifican su idea de que la educación juega un rol fundamental en la constitución de la psique. La relación entre educación y desarrollo psicológico se complejiza cuando se la entiende como una dinámica compleja entre prácticas institucionales asociadas con ideales, valores, intereses y formas de organización socioeconómica y política de una determinada sociedad y entre prácticas concretas en las aulas que dan forma a los actos de enseñar y aprender. Esa relación presenta tensiones, posibilidades de ruptura, retos y oportunidades a la práctica educativa interesada en potenciar las capacidades reflexivas y creativas de los sujetos en las aulas. Los fondos de conocimiento y aprendizaje son conceptos desarrollados desde esta perspectiva y representan un aporte importante a los enfoques socioculturales en educación.

\section{Implicaciones para práctica educativa}

Vygotski elaboró poco el tema de la personalidad y no trabajó los conceptos de subjetividad e identidad. Sin embargo, el conjunto de herramientas representado en los temas contemporáneos examinados en este trabajo ha promovido reflexiones críticas sobre su aplicación a la educación y propiciado la elaboración de nuevos conceptos. Esto tiene obvias implicaciones para la educación. Por un lado, implica prestar atención en las prácticas docentes y en la investigación educativa a aspectos desatendidos por largo tiempo en las instituciones escolares. Por otro lado, implica reenfocar la formación docente para que la atención prestada contribuya positivamente al desarrollo humano. Esa formación debe trascender la orientación a la enseñanza de teorías psicológicas con aplicaciones a los procesos de enseñanza y aprendizaje y al dominio estrategias didácticas cuya efectividad se ponderará en las pruebas de aprovechamiento académico a alumnos y evaluaciones de ejecutoria al personal docente. Debe orientarse a tomar conciencia del alcance de la educación (formal e informal) en la formación de la subjetividad, la identidad y la conciencia. Esta cita de Vygotski resalta la importancia 
del rol de la educación en esos procesos formativos.

...Cuando en realidad la educación, entendida en el más amplio sentido de la palabra, debe ser el eje fundamental alrededor del cual se estructura todo el desarrollo de la personalidad del niño. Una línea dada de desarrollo debe ser entendida como la consecuencia lógica necesaria de una línea de educación. (Vygotski, 1931/1997, p.325).

Las implicaciones educativas de la consideración de las emociones, la afectividad, la subjetividad, las situaciones sociales de desarrollo, la atribución de sentido a las experiencias de aprendizaje vividas en ambientes familiares, escolares y comunitario conminan a una aproximación compleja al sujeto que aprende y los contextos donde lo hace. Estas implicaciones derivan de la exposición que antecede. Dar consideración a los aspectos mencionados exige dejar de ver la escuela meramente como un sistema orientado a la comprensión y reproducción de contenidos disciplinares y formas lineales de pensarlos o un espacio de interacciones sin trascendencia. Exigen introducir en la actividad didáctica experiencias que viabilicen la elaboración de sentidos subjetivos mediante el diálogo, el debate, comunicación de actividades creativas en intercambios que promuevan la expresión de las diferencias y faciliten la comprensión de la diversidad (González Rey, 2009). Esto lleva a una concepción dialógica de la pedagogía (Daniels, 2014).

La consideración de la situación social de desarrollo y las vivencias es central a la motivación y generación de intereses y procesos de aprendizaje significativos y pertinentes a la vida del sujeto. Es en espacios relacionales caracterizados por intercambios dialógico donde se fomenta la participación y la expresión del pensamiento divergente. Las experiencias de aprendizaje deben permitir a cada sujeto la oportunidad de articular sus ideas y emociones y relacionarlas con las de otros. El desarrollo de funciones cognitivas complejas y procesos lógicos no pueden escindirse de la atribución de sentidos subjetivos pues conforman una unidad. Pese a ello, la educación tradicional sigue operando con reglas que perpetúan esa dicotomía y esas reglas se perpetúan en los programas de formación docente. Más aún, como ha señalado Baquero (2007), algunos enfoques contestatarios a las formas tradicionales, como los modelos constructivistas, continuaron en la práctica el énfasis en procesos individuales aun cuando se realicen actividades en grupos y se hable de aprendizaje colaborativo. A su juicio, estos enfoques "han soslayado o soslayan el análisis de la eficacia de contextos de actividad y sus características particulares en la generación de modos específicos de actividad" (p. 87). 
Las intervenciones e investigaciones desarrolladas a partir de los fondos de conocimiento y los fondos de identidad se orientan explícitamente a integrar los contextos de actividad y sus características específicas en la actividad educativa. Los modos de actividad que propicia buscan la implicación activa del sujeto y su cultura vivida en los procesos de aprendizaje para hacerlos significativos. Se ha logrado documentar que al utilizar la variedad de fondos de conocimiento que los estudiantes llevan consigo a las aulas, se validan e incorporan sus identidades en las prácticas escolares. Como la conciencia y la subjetividad, la identidad se construye en interacciones entre el sujeto y el medio histórico, social y cultural en que se desarrolla. Tiene una dimensión individual y una dimensión social que conforman una unidad dentro de la situación en que se manifiestan. Estaban-Guitart y Moll (2014ab) han documentado múltiples cómo ese proceso se observa en las aulas.

Desde la perspectiva histórico-cultural, tanto las actividades de enseñanza e investigación educativas como las de formación docente asumen modelos complejidad para lidiar con las exigencias que las actuales condiciones históricas, sociales y culturales imponen. Las instituciones educativas, desde las que se ocupan de la formación preescolar hasta las que se ocupan de formación a nivel post-doctoral, responden a las sociedades que las albergan y se expresan en relaciones y prácticas sociales concretas. No obstante, hay variaciones en las respuestas. Aunque la mayor parte de ellas van orientadas a la reproducción del sistema social que las origina son impactadas por condiciones histórico-culturales que crean disidencia, resistencias y transformaciones. Algunas de esas transformaciones requieren tiempo y acciones concertadas. Los nuevos desarrollos en el enfoque histórico-cultural pueden servir como herramienta de trabajo hacia la transformación de prácticas educativas. Propongo que el esfuerzo puede intensificarse con herramientas de otras perspectivas afines como la pedagogía crítica (Rodríguez Arocho, 2019) y la psicología crítica (Fleer, González Rey y Jones, en imprenta).

Al discutir algunas implicaciones educativas de la consideración de la subjetividad, González Rey (2016) precisa algunos asuntos que dialogan constructivamente con el reclamo de Baquero (2001) de enfrentar el problema de la sospecha de la educabilidad de sujetos provenientes de sectores marginalizados y con el reclamo de Esteban-Guitart y Moll (2014ab) de potenciar las fortalezas que existen en la diversidad cultural y étnica de los sujetos en contextos escolares. Según González Rey (2016): 
Los procesos educativos de toda institución orientada a la emergencia del individuo como sujeto implican procesos participativos donde los individuos sientan que tienen un espacio social en cual son respetados y apreciados por otros, condición esencial para continuamente generar ante ellos nuevos desafíos que le permitan su desarrollo. Sólo en estas condiciones las diferentes acciones intencionales pueden ser potencialmente efectivas (p. 265).

Muchos documentos de política educativa expresan como ideal la formación de un individuo integral, es decir, que exprese un balance entre lo cognitivo y lo afectivo, entre su desarrollo personal y su desarrollo social. Con frecuencia se habla de un individuo autónomo capaz de tomar decisiones independientes y ejercer libre albedrío. No obstante, muchas de las prácticas educativas representan lo que Paulo Freire identificó como educación bancaria, repetitiva y reproductiva (Rodríguez Arocho, 2019). El formato sigue siendo uno de subordinación a la autoridad del saber dominante en el momento. El formato escolar en el que ese saber se transmite comienza por dejar fuera de las aulas a personas principalmente pertenecientes a grupos que han sido desposeídos de poder y marginalizados y al expulsar de ellas a quienes lo confrontan de diversas maneras.

La incorporación de los temas contemporáneos del enfoque histórico-cultural en la formación docente es un reto cuya superación demanda un paradigma de complejidad que considere el análisis por unidades en lugar del análisis por elementos (Rodríguez Arocho, 2011). En el análisis por unidades no puede separarse el entramado histórico, cultural y social de las actividades que ocurren en las instituciones educativas. Tampoco pueden entenderse aisladamente los componentes del sistema escolar como se hace cuando se atribuye el fracaso escolar a la motivación y limitaciones del estudiante para aprender o al conocimiento y las destrezas del docente para enseñar. La escuela consiste en un conjunto o entramado de relaciones interpersonales situadas y atravesadas por imaginarios sociales, discursos y prácticas la cultura dominante y variadas expresiones de resistencia o cuestionamiento a esa dominación que, muchas veces, busca invalidar la cultura vivida fuera de las aulas.

La formación docente debe trascender los modelos orientados a proveer información y datos concernientes a los saberes disciplinados y el desarrollo de destrezas técnicas asociadas a la didáctica para abarcar, desde una perspectiva reflexiva y crítica, el sujeto y su situación social de desarrollo (Rodríguez Arocho, 2012). Esto implica reconocer variabilidad en los procesos de enseñanza y aprendizaje que, para ser significativos, deben ser significados y 
atribuidos de sentido subjetivo por estudiantes, docentes y otros miembros de la comunidad escolar. La tarea no es fácil pero los desarrollos actuales en el enfoque histórico-cultural ofrecen algunas herramientas para realizarla.

\section{Conclusión}

Los retos que enfrenta la educación contemporánea en tiempos de grandes transformaciones históricas, sociales y culturales son monumentales por su complejidad. En la pasada década una revolución revisionista ha hecho posible la recuperación de aspectos ignorados o minimizados en las interpretaciones dominantes del legado de Vygotski. Este fenómeno, sumado a exámenes críticos de los procesos de producción y uso de conocimiento científico en algunos sectores de la academia internacional, ha generado algunas producciones que pueden servir de herramientas para asumir los retos. Las reflexiones críticas para profundizar en el análisis de los enfoques socioculturales y adelantar su desarrollo y la elaboración de nuevos conceptos que están generando intervenciones e investigaciones guardan la promesa de vencer algunos de los problemas examinados. Temas antes desatendidos como las emociones, la situación social de desarrollo, las vivencias, la subjetividad y la conciencia cobran importancia y sirven como herramientas para el desarrollo de abordajes teóricos y metodológicos más a tono con una perspectiva compleja a todas las dimensiones de la educación como quehacer humano.

\section{Referencias}

Ageyev, V. S. (2003). Vygotsky in the mirror. En Kozulin, A., Gindis, B., Ageyev, V.S. y S. M. Miller, (Eds.), Vygotsky's educational theory in cultural context (pp. 432-449). Cambridge University Press. https://doi.org/10.1017/CBO9780511840975

Álvarez, A. (Ed.). (1997). Hacia un currículum cultural: la vigencia de Vygotski en la escuela. Fundación Infancia y Aprendizaje.

Álvarez, A. y del Río, P. (2007). Una introducción a las dos psicologías de Vygotski. En P. del Río y A. Álvarez (Eds.). Escritos sobre arte y educación creativa de L.S. Vygotski (pp. 7-20). Fundación Infancia y Aprendizaje.

Bakhurst, D. (2007). Vygotsky's demons. In H. Daniels, M. Cole y J.V Werstch (Eds.), The Cambridge companion to Vygotsky (pp.50-76). Cambridge University Press. https://doi.org/10.1017/CCOL0521831040

Baquero, R. (2001). La educabilidad bajo sospecha. Cuadernos de Pedagogía Rosario, 4(9), 7185.

Baquero, R. (2003). La educabilidad como problema político: Una mirada desde la psicología educacional. Documento de Trabajo No. 9. http://hdl.handle.net/10908/772 
Baquero, R. (2006). Sujetos y aprendizaje. Ministerio de Educación, Ciencia y Tecnología.

Baquero, R. (2009). Zona de desarrollo próximo, sujeto y situación: El problema de las unidades de análisis en la psicología educativa. Actualidades Investigativas en Educación, 9 (Número especial), 1-25. DOI 10.15517/AIE.V9I4.9514

Baquero, R. (2017). Desarrollo subjetivo, prácticas educativas y prácticas escolares. Obutchénie, 1(2), 291-309. DOI: http://dx.doi.org/10.14393/OBv1n2a2017-3

Blanck, G. (2001). Prefacio. L.S. Vygotski, Psicología pedagógica: un curso breve. Aique.

Chaklin, Seth (2003). The zone of proximal development in Vygotsky's analysis of learning and instruction. En A. Kozulin, B. Gindis, V.S. Agueyev y S.M. Miller (Eds.). Vygotsky's educational theory in cultural context (pp. 39-64). Cambridge University Press. https://doi.org/10.1017/CBO9780511840975.004

Dafermos, M. (2016). Critical reflection on the reception of Vygotsky's theory in the international academic community. Cultural-Historical Psychology, 12(3), 27-43.

https://doi.org/10.17759/chp.2016120303

Dafermos, M. (2018). Rethinking cultural-historical psychology: a dialectical perspective to Vygotsky. Springer. DOI 10.1007/978-981-13-0191-9

Daniels, H. (Ed.) (1993). Charting the agenda: educational activity after Vygtotsky. Routledge.

DOI: $10.1207 / \mathrm{s} 15327884 \mathrm{mca} 0401 \_9$

Daniels, H. (2001). Vygotsky and pedagogy. Routledge.

Daniels, H. (2014) Vygotsky and dialectical pedagogy. Cultural-Historical Psychology, 10(3), $19-29$.

del Río, P. y Álvarez, A. (2007). La psicología del arte en la psicología de Vygotski. En P. del Río y A. Álvarez (Eds). L.S Vygotski. La tragedia de Hamlet. Psicología del arte (p. 738). Fundación Infancia y Aprendizaje.

Elhammoumi, M. (2001). Lost-Or merely domesticated? The boom in socio-historicocultural theory emphasizes some concepts, overlooks others. En S. Chaklin (Ed.), The theory and practice of cultural-historical psychology (pp. 200-217). Arhaus University Press.

Esteban-Guitart, M. y Moll, L. (2014a). Funds of Identity: A new concept based on the funds of knowledge approach. Culture and Psychology, 20 (1), 31-48. DOI: $10.1177 / 1354067 X 13515934$

Esteban-Guitart, M. y Moll, L. (2014b). Live experience, funds of identity and education. Culture and Psychology, 20 (1), 31-48. DOI: 10.1177/1354067X13515940

Esteban-Guitart, M. (2016). Funds of identity: Connecting meaningful learning experiences in and out of school (Learning in doing social, cognitive and computational perspectives). Cambridge University Press. DOI: 10.1017/CBO9781316544884

Fleer, M., González Rey, F. \& Veresov, N. (Eds.) (2017). Perezhivanie, emotions and subjectivity: Advancing Vygotsky's legacy. Springer. https://doi.org/10.1007/978-981-104534-9 
Fleer, M., González Rey, F. y Jones, P. (en imprenta). Cultural-Historical and critical psychology: Common grounds, divergences and future pathways. Springer.

García, L. N. (2019). On Vygotsky's international celebration, or how to critically appropriate authors from the past. En A. Yasnitsky (Ed.), Questioning Vygotsky's legacy (pp. 161-183). New York, NY: Routledge.

González Rey, F. L. (2002). Sujeto y subjetividad: Una aproximación histórico-cultural. Thompson.

González Rey, F. (2007). Investigación cualitativa y subjetividad. McGraw Hill

González Rey, F.L (2009). Epistemología y ontología: Un debate necesario para la psicología de hoy. Diversitas: Perspectivas en Psicología, 5(2), 205-224.

González Rey, F. L. (2011a). El pensamiento de Vygotsky: contradicciones, desdoblamientos y desarrollos. Trillas. DOI: 10.18774/448x.2016.13.278

González Rey, F. L (2011b). The path to subjectivity: Advancing alternative understanding of Vygotsky and the cultural-historical legacy. En P. Portes y S. Salas (Eds.), Vygotsky in the 21 st century society: Advances in cultural-historical theory and praxis with non-dominant communities 9PP. 32-49). Peter Lang.

González Rey, F.L. (2016). Psicología en la educación: Implicaciones de la subjetividad en una perspectiva cultural-histórica. Revista Puertorriqueña de Psicología, 27 (2), 260-274.

González Rey, F. L. (2017). Advances in subjectivity from a cultural-historical perspective: Unfolding and consequences for cultural studies today. En M. Fleer, F.L. González Rey y N. Veresov (Eds.). Perezhivanie, emotions and subjectivity: Advancing Vygtosky's legacy (pp. 173-193). Springer. DOI: 10.1007/978-981-10-4534-9_9

González Rey, F.L. (2019a): Fifty years after L. I. Bozhovich's personality and its formation in childhood: recovering her legacy and her historical role. Mind, Culture, and Activity, 26(2), 108-120. DOI: 10.1080/10749039.2019.1616210

González Rey, F. L. (2019). Subjectivity as a new theoretical, epistemological, and methodological pathway within cultural-historical psychology. En F. L. González Rey, A. Mitjáns Martínez y D. Magalhães Goulart (Eds.), Subjectivity within the culturalhistorical approach (pp.21-36). Springer. DOI:https://doi.org/10.1007/978-981-13$\underline{3155-8 \_2}$

González, N., Moll, L. y Amanti, C. (2005). Funds of knowledge: Theorizing practices in households, communities and classrooms. Lawrence Erlbaum Associates Publishers.

González Rey, F.L y Mitjáns Martínez, A. (2016). Perezhivanie: Advancing in its implications for the cultural-historical approach. International Research in Early Childhood Education, 7(1), 142-160. https://doi.org/10.4225/03/5810026e807e2

González Rey, F. L. y Mitjáns Martínez, A. (2019). The constructive-interpretative methodological approach: Orienting research and practice on the basis of subjectivity. En F. L. González Rey, A. Mitjáns Martínez y D. Magalhães Goulart (Eds.), Subjectivity within the cultural-historical approach (pp.37-60). Springer. https://doi.org/10.1007/978-981-13-3155-8_3 
González Rey, F.L., Mitjáns Martínez, A. \& Magalhães Goulart, D. (Eds.) (2019). Subjectivity within the cultural-historical approach. Springer. DOI: 10.1007/978-981-13-3155-8_1

Karpov, Y. V. (2014). Vygotsky for educators. Cambridge University Press. https://doi.org/10.1017/CBO9781107588318

Kozulin, A. (1998). Psychological tools. Harvard University Press.

Kozulin, A. Gindis, B., Ageyev, V.S. y Miller, S.M. (Eds.). Vygotsky's educational theory in cultural context. Cambridge University Press. https://doi.org/10.1017/CBO9780511840975

Matusov, E. (2008). Applying sociocultural approach to Vygotskian academia: Our tzar it is not like yours and yours isn't like ours. Culture and Psychology, 14(1): 5-35. DOI: $\underline{10.1177 / 1354067 X 07085808}$

Mok, N. (2017). On the concept of perezhivanie: A quest for a critical review. En M. Fleer, F.L. González Rey y N. Veresov (Eds.), Perezhivanie, emotions and subjectivity: Advancing Vygtosky's legacy (pp. 19-46). Springer.

Moll, L. (Ed.) (1990). Vygotsky and education: Instructional implications and applications of sociohistorical psychology. Cambridge University Press.

https://doi.org/10.1017/CBO9781139173674

Moll, L. (1997), Vygotski, la educación y la cultura en acción. En A. Álvarez (Ed.), Hacia un currículum cultural: La vivencia de Vygotski en la escuela (pp. 39-53). Fundación Infancia y Aprendizaje.

Moll, L. (2011). Only life educates: Immigrant families, the cultivation of biliteracy and the mobility of knowledge. En P. Portes \& S. Salas (Eds.), Vygotsky in the 21st century society: Advances in cultural-historical theory and praxis with non-dominant communities (pp. 152-161). Peter Lang.

Moll, L. (2014). L.S. Vygotsky and education. Taylor and Francis. DOI: $10.4324 / 9780203156773$

Rivière, A. (1984). La psicología de Vygotski. Aprendizaje Visor.

\section{DOI: $10.1080 / 02103702.1984 .10822043$}

Rodriguez Arocho, W. C. (2000). Una aproximación histórico cultural a la revolución cognoscitiva de cara al nuevo milenio. Educare, (8), 22-20.

Rodríguez Arocho, W. C. (2010). Los conceptos de vivencia y situación social del desarrollo: Reflexión en torno a su lugar en el modelo teórico de Lev. S. Vygotski. En S. Aburto Morales \& C. Meza Peña (Eds.), Tutoría para el Desarrollo Humano: Enfoques (pp. 7392). Universidad de Nuevo León.

Rodríguez Arocho, W. C. (2011). El enfoque histórico-cultural como marco conceptual para la investigación educativa. Paradigma, 30(1), pp. 33-62.

Rodríguez Arocho, W. C. (2012). La formación docente: Su resignificación desde el enfoque histórico-cultural. Pedagogía, 46(1), 9-18. 
Rodríguez Arocho, W. C. (2013). El lugar de la afectividad en la psicología de Vygotski: Reflexividad histórica y reivindicación. Propósitos y Representaciones, 1(2), 105-129.

Rodríguez Arocho, W. C. (2015). Reflexividad histórica, problematización e indagación dialógica como herramientas para repensar el concepto vygotskiano de zona de desarrollo próximo. Revista Puertorriqueña de Psicología, 26(1), 10-24.

Rodríguez Arocho, W. C. (2018). Tecnologías de la información y la comunicación: Nuevas configuraciones mentales y sus implicaciones para la educación. Revista de Psicología, 27(1), 1-12. Recuperado de https://revistapsicologia.uchile.cl/index.php/RDP/article/view/50751

Vélez-Ibañez, C.G. y Greenberg, J. B. (1992). The formation and transformation of funds of knowledge. Anthropology and Education Quarterly, 23 (4), 313-335.

Veresov, N. (1999). Undiscovered Vygtosky. Peter Lang.

Veresov, N. (2017) The concept of perezhivanie in cultural-historical theory: content and contexts. .En M. Fleer, F.L. González Rey y N. Veresov (Eds.). Perezhivanie, emotions and subjectivity: Advancing Vygtosky's legacy (pp. 47-70). Springer.

Vygotski, L.S. (1925/1991). La conciencia como problema en la psicología del comportamiento. En L. S. Vygotski Obras Escogidas, Tomo I (pp. 39-60). Madrid: Aprendizaje Visor.

Vygotski, L. S. (1926/2001). L.S. Vygotski, Psicología pedagógica: un curso breve. Aique.

Vygotski, L. S. (1927/1991). El significado histórico de la crisis de la psicología. En L. S. Vygotski: Obras Escogidas, Tomo I (pp. 275-413). Madrid: Aprendizaje Visor.

Vygotski, L.S. (1931/1995). Historia del desarrollo de las funciones psíquicas superiores. En L. S. Vygotski Obras Escogidas, Tomo III (pp. 11-340). Madrid: Aprendizaje Visor.

Vygotski, L. S. (1932/1996). El problema de la edad. En L. S. Vygotski: Obras Escogidas, Tomo IV. (pp. 251-273). Madrid: Aprendizaje Visor.

Vygotski, L. S. (1933/1996). La crisis de los siete años. En L. S. Vygotski: Obras Escogidas, Tomo IV. (pp.377-412). Madrid. Aprendizaje Visor.

Vygotski, L.S. (1934/1993). Pensamiento y lenguaje. En Lev S. Vygotsky: Obras Escogidas, Tomo II, (pp. 9-348). Madrid. Aprendizaje Visor.

Vygotski, L.S. (1935a/2001). Aprendizaje y desarrollo intelectual en la edad escolar. En M. Cecchini (Ed.), Luria, Leontiev, Vygotsky: Psicología y pedagogía (pp.23-39). Akal.

Vygotski, L. S. (1935b/2004). Teoría de las emociones: Estudio histórico-psicológico. Akal.

Vygotski, L. S. (1935c/1994). The problem of the environment. En R. Van der Veer y J. Valsiner (Eds.), The Vygotsky reader (pp.338-354). Blackwell.

Yasnitsky, A. (2010) Guest Editor's Introduction. Journal of Russian \& East European Psychology, 48:1, 3-13 DOI: 10.2753/RP01061-0405480100

Yasnitsky, A. \& Van der Veer, R. (Eds.) (2016). Revisionist revolution in Vygotsky studies. Routledge. DOI:10.4324/9781315714240 
Zavershneva, E. (2010a). The Vygotsky family archive: New findings. Notebooks, notes and scientific journal of L.S. Vygotsky (1912-1934). Journal of Russian and East European Psychology, 48(1), 34-60. DOI: 10.2753/RPO1061-0405480102

Zavershneva, E. (2010b). The way to freedom. Journal of Russian and East European Psychology, 48(1), 61-90. DOI: 10.2753/RPO1061-0405480103

Zavershneva, E. \& Van der Veer, R. (Eds.) (2018). Vygotsky's notebooks: a selection. Springer. 